\title{
GCU
}

Glasgow Caledonian

University

University for the Common Good

\section{Barriers and facilitators to implementing functional family therapy in a community setting: client and practitioner perspectives}

McPherson, Kerri E.; Kerr, Susan; Casey, Beth; Marshall, John

Published in:

Journal of Marital and Family Therapy

DOI:

10.1111/jmft.12221

Publication date:

2017

Document Version

Author accepted manuscript

Link to publication in ResearchOnline

Citation for published version (Harvard):

McPherson, KE, Kerr, S, Casey, B \& Marshall, J 2017, 'Barriers and facilitators to implementing functional family therapy in a community setting: client and practitioner perspectives', Journal of Marital and Family Therapy, vol. 43, no. 4, pp. 717-732. https://doi.org/10.1111/jmft.12221

\section{General rights}

Copyright and moral rights for the publications made accessible in the public portal are retained by the authors and/or other copyright owners and it is a condition of accessing publications that users recognise and abide by the legal requirements associated with these rights.

Take down policy

If you believe that this document breaches copyright please view our takedown policy at https://edshare.gcu.ac.uk/id/eprint/5179 for details of how to contact us. 
BARRIERS AND FACILITATORS TO IMPLEMENTING FUNCTIONAL FAMILY THERAPY IN A COMMUNITY SETTING: CLIENT AND PRACTITIONER PERSPECTIVES

Kerri E. McPherson, Susan Kerr, Beth Casey

Glasgow Caledonian University, UK

John Marshall

NHS Greater Glasgow and Clyde, UK

Dr Kerri McPherson, $\mathrm{PhD}$ is a Reader in the Institute for Applied Health Research at Glasgow Caledonian University, Scotland.

Dr Susan Kerr, PhD is a Reader in the Institute for Applied Health Research at Glasgow Caledonian University, Scotland.

Dr Beth Casey, PhD was a Research Assistant in the Institute for Applied Health Research at Glasgow Caledonian University, Scotland during the time the research was conducted.

Dr John Marshall is a Consultant Forensic \& Clinical Psychologist for NHS Greater Glasgow and Clyde NHS.

This research is part of a larger study that has been presented to the funding body in the form of a final report. Members of the research team had no formal or financial association with Functional Family Therapy LLC. The team wishes to acknowledge the contribution made to the research design and conduct by Drs Josie Williams and Stella Bain and Mr Steve Collins and Ms Pamela McDaid. 
Corresponding author: Dr Kerri McPherson, Institute for Applied Health Research, Glasgow Caledonian University, Cowcaddens Road, Glasgow, UK G4 0BA.

Kerri.mcpherson@gcu.ac.uk 


\begin{abstract}
While Functional Family Therapy (FFT) is known to be effective in addressing adolescent behavioral problems, there has been little exploration of issues relevant to its transport from the tightly controlled setting of clinical trials into routine service delivery. This study sought the views of key stakeholders, clients and practitioners, on barriers and facilitators to the successful implementation of FFT. Undertaken in a community setting in Scotland, interviews were carried out with 12 adolescents, 14 parents/caregivers and 6 practitioners. Results focus on: Referral process and pre-intervention contact; Engagement of families; Structure and delivery; Organizational factors. Although barriers to engagement were identified, FFT was viewed as an acceptable, appropriate and feasible intervention with the potential to improve adolescent wellbeing in 'real-world' settings.
\end{abstract}




\section{BARRIERS AND FACILITATORS TO IMPLEMENTING FUNCTIONAL FAMILY THERAPY IN A COMMUNITY SETTING: CLIENT AND PRACTITIONER PERSPECTIVES}

In recent years, global public health policy has focused attention on adolescence (i.e., 10-19 years) as a key life stage. Early childhood experiences are known to impact on the health and wellbeing of adolescents and, in turn, health and wellbeing in adolescence impacts on the adult years, with the potential for intergenerational transmission both of assets and deficits (World Health Organization, 2014). Effective interventions in adolescence are, therefore, crucial in addressing problems that have arisen in the first decade of life, in preventing problems later in the life-course, and in ensuring the best outcomes for future generations (Patten et al., 2014; World Health Organization, 2014).

When considering the health and wellbeing of adolescents, mental health has been highlighted as a priority for policy makers and practitioners across different sectors, including public health, social care and education (e.g., Brauner \& Stephens, 2006). Externalizing behaviors, including conduct disorders, are the most common form of mental health problem in children and adolescents and are characterized by repetitive and persistent patterns of antisocial, aggressive or defiant behavior that exceed age-appropriate expectations (American Psychiatric Association, 2013; NICE, 2013). Estimates suggest that 5-10\% of children and adolescents exhibit externalizing behaviors that are clinically significant and persistent in duration (Tonge, 2007; Hill, 2002). Importantly, externalizing behaviors are known to resonate across a young person's life, affecting attendance at school, educational achievement and future health and life chances (Liu, 2004; Moffit, Caspi, Harrington, \& Milne, 2002; Rapport et al., 2002). Adolescents experiencing externalizing behavioral problems are also more likely to come into contact with the youth/criminal justice system (Brauner \& Stephens, 2006). 
While it is acknowledged that factors associated with the onset and maintenance of externalizing behaviors are multiple and inter-related, existing at the level of the individual, family, community (e.g., school and peer groups) and society (Liu, 2004), the role of the family is considered to be central (Price, Chiapa, \& Walsh, 2013). Review-level evidence demonstrates that parent-child relationships characterized by positive communication, feelings of nurturance, support and low levels of conflict are protective, while poor communication, low levels of support and high levels of conflict increase vulnerability (McPherson et al., 2014). The importance of the wider family has also been highlighted, with levels of cohesiveness, perceptions of justice (i.e., fairness) trust and time spent together impacting behavioral outcomes (McPherson et al., 2014).

Given evidence on the important role of family, a range of interventions, both preventative and treatment-focused, have been developed that aim to harness the family system to affect positive outcomes for adolescents. Functional Family Therapy (FFT) (Alexander, Waldron, Robbins, \& Neeb, 2013) is one such family-focused treatment intervention for adolescents with externalizing behavioral problems (Alexander \& Parsons, 1973; Littell Winsvold, Bjørndal, \& Hammerstrøm, 2007). First developed in the US in the 1960s, FFT is underpinned by a theoretical assumption that problem behaviors exhibited by adolescents are rooted in dysfunctional family relations (see Robbins, Alexander, Turner \& Hollimon [2016] for further information about the evolution of FFT). The intervention aims to strengthen protective factors (e.g., parenting skills), reduce risk factors (e.g., negativity and blame) and improve communication among family members (Alexander et al., 2013; Breuk et al., 2006). FFT is manualized and is delivered by specially trained practitioners over a threemonth period in five phases: 1) Engagement 2) Motivation, 3) Relational Assessment 4) Behavior Change, and 5) Generalization (Alexander, 2011; see Alexander et al. [2013] for further information about FFT). The final phase goal is the generalization of skills to other 
social and community interventions/settings, such as parenting classes or school clubs (Alexander et al., 2013; Littell et al., 2007).

Although individual studies show different levels of effect, recent meta-analyses, reviews of reviews and cost-benefit analyses demonstrate the effectiveness and costeffectiveness of FFT across a number of outcomes. These include reducing externalizing behaviors (e.g., delinquency), criminal behavior and substance misuse (Casey, McPherson, \& Kerr, 2016; Washingston State Institute for Public Policy, 2016; Stratton, 2005; Woolfenden, Williams, \& Peat, 2002). In addition, alongside other forms of systemic family therapy, FFT has demonstrated better outcomes than other types of psychotherapy across a number of different domains (Baldwin, Christian, Berkeljon, \& Shadish, 2012; von Sydow, Retzlaff, Beher, Haun, \& Schweitzer, 2013). Consequently, FFT is listed as an evidence-based program on the US-based Blueprints for Healthy Youth Development registry (http://blueprintsprograms.com) and it is recommended by the UK National Institute for Health and Care Excellence (NICE, 2011).

\section{Implementation of evidence-based programs}

Successful transport of evidence-based programs from the tightly controlled setting of a clinical trial to routine service provision is known to be contingent, at least in part, upon factors linked to the implementation process (Proctor et al., 2011). For example, therapist adherence to the FFT model (Graham et al., 2014; Sexton \& Turner, 2010) and levels of family engagement, assessed as treatment completion (Graham et al., 2014; Sholevar, Baron, Aussetts \& Spiga, 2010), have been shown to impact on outcomes following intervention. However, while it has been possible to identify implementation factors that influence the relationship between FFT and outcome, there is very limited evidence available to explain why issues such as family engagement may be sub-optimal (Casey et al., 2016). 
To better understand this, the implementation science literature advocates taking account of stakeholder views on the acceptability, appropriateness and feasibility of the intervention (Proctor et al., 2011). A number of articles have described the implementation of FFT from an organizational perspective (e.g., Breuk et al., 2006; Zazzali, Sherbourne, Hoagwood, Greene, Bigley, \& Sexton, 2008) and have highlighted factors that support implementation (e.g, organizational culture); however, it remains unclear whether other stakeholder groups have similar views. Particularly important in this respect are the views of the people delivering the intervention to families (FFT practitioners) and the families in receipt of it.

In the only study to date to capture the views of intervention recipients, Celinska and colleagues (Celinska, Cheng, \& Virgil, 2015) focused on family members' levels of satisfaction with FFT. Interestingly, both adolescents and parents reported satisfaction with the intervention, but parents were shown to be more satisfied than their children. While Celinska's study makes a useful contribution to the evidence base, the focus on satisfaction alone means that the study is limited in terms of the contribution it can make to understanding how families experience their participation in FFT and it sheds no light on the views and experiences of other stakeholders, including practitioners who deliver the intervention.

The lack of information on FFT practitioners' experiences of implementation represents a significant gap in the literature. Successful transport of an efficacious intervention to a service delivery context is contingent upon the intervention and the implementation process being feasible and acceptable to all stakeholders, including the workforce involved in its delivery and service users (Fixsen, Blase, Duda, Naoom, \& Van Dyke, 2010; Weisz \& Kazdin, 2010). Moreover, as demonstrated in the study undertaken by Celinska (Celinska et al., 2015), there is evidence that different groups of stakeholders may present different accounts when asked to share their views on a particular intervention, and these differences 
may be polarized when comparing the views of those who deliver interventions with those in receipt of them.

The current study was designed to address this important gap in the literature by exploring the views and experiences of two key stakeholder groups on the implementation of FFT. Our aim was to identify key barriers and facilitators to successful implementation. Specifically, we sought to triangulate the views of those delivering the intervention, FFT practitioners, with clients in receipt of it, adolescents and their parents/caregivers. Evidence from the extant implementation science literature (Fixsen, Naoom, Blase \& Friedman, 2005) informed the data collection and analysis processes. Areas of focus included: factors relating to family engagement with the intervention, such as the referral process, the delivery format and the service user population; core implementation factors, such as the training and supervision of providers; and organizational factors, such as the organizational culture, and factors relating to the professional practitioner workforce.

\section{METHODS}

\section{Study setting}

The study was carried out in the west of Scotland within a community-based FFT service. The practitioners were trained as a group by the purveyor organization FFT LLC (http://www.fftllc.com), using their Clinical Training Manual (Alexander, 2011). At the time of data collection, the team had been delivering FFT to families in the local community for approximately 18 months. FFT was offered to families on a voluntary basis rather than being mandated.

\section{Research approach}

A qualitative approach was adopted, guided by a critical realist perspective (McEvoy \& Richards, 2003), which views social reality as multi-layered (Baskar, 1989). Critical realists propose that intervention mechanisms of action (e.g., how an intervention such as FFT works) 
may not be observable but can be identified through their effects (Houston, 2001) and that people's views and experiences are influenced by both psychological and social processes (Houston, 2001). Thus, the views and experiences of practitioners and clients who participated in this study were considered in the context of underlying generative mechanisms that include a matrix of individual, organizational and socio-cultural factors (Oliver, 2012).

The study was approved by Glasgow Caledonian University Ethics Committee and by the appropriate local government authority in Scotland.

\section{Participants and recruitment}

Adolescents and parents/caregivers. Given the aim of the study was focused on understanding the views and experiences of FFT, it was considered important and appropriate that adolescent and parent/caregiver participants had completed the full intervention, irrespective of outcome. A total of 75 families had completed FFT at the time of recruitment and were eligible to participate in the study. Purposive sampling was used to recruit families. The intention had been to stratify the sample on the basis of their primary reason for referral to FFT but, as might be expected in a community-based service, for most adolescents the reasons were multiple and inter-related with no primary reason identified. The target sample was, therefore, stratified only by age (11-13 years and 14-17 years) and sex (male and female) with the aim of recruiting 16 adolescents and their families. This sampling approach ensured representation in each strata and was used as it was believed that the age and sex of the adolescent may impact on the views and experiences of the participants. When adolescents had been identified their parent/caregiver(s) were also invited to participate.

The family circumstances of each adolescent who met the inclusion criteria were reviewed by the FFT service team prior to contact by the researcher. Families who met the inclusion criteria but were considered to be 'high risk' in terms of safety of the researcher or 'too vulnerable' to participate were not contacted about the study $(n=8)$. Families identified 
as being appropriate $(n=67)$ were contacted by the FFT team and provided with an information pack that included an information sheet and invitation to participate. Those who expressed an interest in participating were visited at home by the researcher prior to obtaining consent. It was emphasized to the adolescents and parents/caregivers that participation was voluntary and their choice in relation to this would not affect future service provision. In total 12 adolescents (six males and six females) and 14 parents/caregivers, from 13 different families, were recruited. All of the parents were mothers $(n=11)$ and all of the non-parent caregivers $(n=3)$ were grandmothers. The families lived in areas high in indicators of deprivation.

FFT practitioners. Practitioners were recruited from the community-based FFT team, which comprised one manager and six FFT practitioners at the time. Given the relatively small number of staff, all were provided with an information sheet and consent form. All six of the FFT practitioners agreed to participate.

\section{Data collection}

Semi-structured one-to-one interviews were carried out with adolescents and their parent/caregiver(s) in the family home. The interviews were conducted separately as it was acknowledged that the adolescent and their parent/caregiver(s) may have differing views and experiences of FFT that they may not wish to discuss in each other's presence. Individual semi-structured interviews with the FFT practitioners were conducted at their place of work. Individual, rather than a group, interviews were conducted to allow the practitioners to discuss issues they may have considered sensitive or confidential.

Interview schedules, tailored to each of the participant groups, were used to focus the data collection process. The content of these was informed by the aims and theoretical underpinnings of the study (critical realism) and the literature on implementation science. In addition, the schedule for the families was prepared taking into account the age of participants 
and the potential sensitivity of what was being explored (e.g., behavioral issues that brought the adolescents into contact with the service). The schedule for the practitioners included a line of questioning in relation to organizational factors. It was recognized that unequal power relations exist between an interviewer and study participants so the schedule was also designed to encourage the participants to share their own views and to comment on issues they believed to be relevant. Prompts were used to encourage detailed responses.

The interviews were audio-recorded with permission. If participants did not wish the interview to be recorded, the researcher took hand-written notes. In order to check for accuracy, the researcher read back these written notes to the participant following the completion of the interview. Participants were advised on a number of occasions about the purpose of the study, the boundaries with respect to confidentiality and of their right to withdraw. All participating adolescents and parents/caregivers received a $£ 10$ gift voucher to thank them for their time. The FFT practitioners did not receive a gratitude payment as the interviews took place during their hours of employment.

\section{Data analysis}

The audio-recorded interviews and notes were transcribed verbatim and checked for accuracy. Names and any identifying features were removed. The data were then analyzed thematically, with the assistance of NVivo v10 (2012), using the process described by Braun and Clarke (2006). The step-by-step process involved: familiarization with the data (i.e., reading and re-reading of the transcripts), coding, building categories, and the generation of themes. The initial analysis of the data was undertaken by BC. To ensure rigor in the process the emerging findings were discussed regularly among members of the research team with consensus being reached. 


\section{RESULTS}

As discussed the data collection and analysis processes were informed by the extant literature on implementation science (Fixsen et al., 2005). The findings are, therefore, presented under four broad categories: Referral process and pre-intervention contact; Engagement of families; Structure and delivery; and, Organizational factors. The key themes that emerged under each of these headings are presented below, with the 'discussion' of the findings in the next section. The accounts from the families and practitioners have been integrated where appropriate and similarities and differences in the views and experiences between the different groups are highlighted.

\section{Referral process and pre-intervention contact}

Key themes to emerge in this area were: Knowledge and understanding; Feeling pressured; and, First steps in relationship building. Each is discussed below.

Knowledge and understanding. There was recognition from both the practitioners delivering the intervention, and the families in receipt of it, that the referral process and preintervention contact were key points in the FFT journey. Practitioners recognized their role in increasing awareness, knowledge and understanding of the FFT service to referring agencies and there was a sense that, at times, they had to work to reduce skepticism amongst some external colleagues.

I think [for] some social workers it may just 'oh another model' ... we need to ... help social workers understand about Functional Family Therapy and how beneficial it is. (Practitioner 2)

The majority of the practitioners felt that families would be more open to participating in the intervention if their social worker had explained accurately what FFT involved and provided a rationale for the referral. 
You're hoping ... that when you initially go out for that initial home visit with the social worker that the family do know a little bit about it.

(Practitioner 6)

While some young people struggled to remember details of the referral process, those who did, and the majority of parents/grandparents, recalled it had been made by the family's social worker. Importantly, they described it as having been explained as being recommended for their individual and/or family issues, including problems with relationships.

My social worker thought it might be useful as a family. Yes, because of my temper and things she thought it might be useful. (Family 12: Adolescent, Female, 15-16y)

I was referred by... my social worker ... Due to ma parental skills. I couldn't put down firm boundaries with [my son] and keep to them. (Family 7: Parent)

Although most families appeared to have been provided with sufficient information to understand FFT and what it sought to achieve, a few felt that the referring individuals weren't always fully informed.

I don't think the social worker really understood that much about it ... Cause it was quite new I think ... (Family 3: Parent)

Feeling pressured. The premise of the service was that family participation in FFT should be voluntary; however, the practitioners noted that some families felt pressured into participating and they saw this as a potential barrier to subsequent engagement.

I think the ones who [feel] sort of coerced into it will be there for the appointments, but aren't really fully engaged in the process ... or else they will develop this pattern where they'll attend for one appointment then cancel ... so you've never really got that engagement (Practitioner 3) 
The families themselves confirmed that participation was sometimes part of a recommendation by their social worker and they felt it was linked to court proceedings.

Ah ... didn't really have a choice in the matter (laughs). (Family 10:

Adolescent, Female, 15-16y)

While it could be argued that there is likely to be a difference between wanting to engage and mandatory participation, interestingly, some of those who felt pressured into participating in the intervention also reported that they could see the potential benefit of being referred. Essentially, there was an external compulsion but also an individual willingness to engage.

It was something I had to do. [But I was also] wantin' to do it. (Family 5:

Adolescent, Male, 13-14y)

First steps in relationship building. In addition to what was discussed at the point of referral, the practitioners described the first visit to families as an important facilitator of engagement. It was an opportunity to assess the appropriateness of the referral and the individual family's willingness to take part. It also gave practitioners time to explain the rationale for FFT and was viewed as an important first step in building a relationship with families.

I always try to go through that whole rationale with them, to check whether they're thinking this is the right thing for them as well. (Practitioner 4)

Families who could remember the first visit reiterated these points; however, what seemed most important was the approach and manner of the FFT practitioner. The practitioners were frequently described as non-authoritative and personable.

[FFT practitioner] treated us as people rather than a job ... she was nice and warm and friendly. (Family 3: Adolescent, Female, age missing) 
We felt quite comfortable with her ... she made you feel at ease. (Family 4:

Grandparent)

In addition, importance was placed on the FFT practitioner using appropriate language to enable understanding, and emphasizing working in partnership with the family rather than 'dictating' what would happen.

It wasn't ... 'this is gonna happen, this is our action plan'. She [FFT

Practitioner] came and introduced [herself] and [said] this is what I do, this is what I can offer. And do you need the help? So [we said] yes we need the help. (Family 8: Parent)

\section{Engagement of families with the intervention}

The themes that emerged in this area were: Trust and honesty; Seeing the need and experiencing change; Views of significant others; and, Psycho-social and cultural influences.

Trust and honesty. Both groups of participants spoke about the quality of the on-going relationship between the family and practitioner as crucial to facilitating engagement and intervention success. The qualities required in the FFT practitioners and the approach taken were described by the participants as:

Good listening skills ... warmth and active listening... being able to empathize with people. (Practitioner 6)

She just wanted to ... get to know us better... (Family 2: Adolescent, Female, age missing)

It's about how you can put that knowledge across ... the ability to make people feel comfortable. (Family 3: Parent) 
These qualities were perceived as promoting trust in the FFT practitioners, which enabled family members to open up during sessions.

I was comfortable sitting with [FFT practitioner] and being honest and stuff like that... [FFT practitioner] was just very open ... (Family 10: Adolescent, Female, 15-16y)

Be honest... gets ye in the right tracks... ye can tell them stuff, ye can trust [them].' (Family 9: Adolescent, Female, 15-16y)

Linked to this, some parents emphasized the distinction between FFT and more top-down, expert-led interventions. They indicated that they engaged with FFT as a result of being respected as individuals, with their problems being listened to and taken seriously.

Rather than them ... sayin 'right, there's the book, read the book'... when [FFT practitioner] came out he explained things ... They would have tae come oot an' listen tae the family's problems first. (Family 7: Parent)

Indeed, practitioners stressed the importance of a balanced alliance in the relationship, not apportioning blame or taking sides with individual family members. Most felt that a strengths-based approach facilitated successful outcomes with families. This was articulated as increasing the hopefulness of the family, identifying and encouraging strengths, praising progress and positive change, communicating a belief that the family were able to make changes themselves. A few noted that being honest and open with families was an important aspect of engagement, particularly in relation to safeguarding concerns.

Being open about what you're doing, why you're doing it, erm you know that there's nothing that you'd be hiding from them... if there are risks you would inform them you do need to take it back to social work or whatever.

(Practitioner 3) 
Some of the young people and parents also spoke about practitioners' honesty in relation to problems they might be experiencing, feeling that it was done in a non-judgmental and caring manner. This honesty was coupled with a view from families that FFT practitioners were reliable.

The [FFT practitioner] never cancelled meetings and always kept to the times arranged. (Family 1: Adolescent, Male, 15-16y).

Moreover, this reliability extended out with the face-to-face sessions and the opportunity to contact the practitioner for advice at other times being welcomed.

I just felt if I had any problems and I couldn't get social work or anybody I could still phone [FFT practitioner] and ... [they] would help with [son's] college placement and like phoning up on behalf of me ... I can still even to this day, phone [FFT practitioner]... for advice. (Family 11: Parent)

Seeing the need and experiencing change. In addition to describing factors that facilitated engagement, practitioners described the need to be persistent and patient when families were hard to engage, disengaging or not making progress. Strategies included maintaining drive, energy and momentum. They spoke about being persistent in contacting the family and being approachable in-between sessions, maintaining an understanding and non-judgmental attitude and providing a strong rationale for continued involvement in the intervention.

A number of individual and family factors were noted as facilitating engagement. Practitioners thought that families who recognized they were experiencing problems and who were motivated to change engaged well with FFT. This was supported by the experience of families, some of whom described a need for support.

Me and [young person] decided... my husband decided... it couldn't certainly at that point in our lives have done any harm, so we may as well go ahead and 
see what we had to do ... we were at a point when [young person] was

possibly going to be going into care. (Family 10: Parent)

Furthermore, experiencing positive changes, such as improved parenting skills, better communication and spending time together as a family, was associated with continued engagement.

Just every session it was getting better and it helped ... Helped me and mum to. Realized it's got me and mum closer. (Family 7: Adolescent, Male, 13$14 \mathrm{y})$

Seeing an improvement helped me keep going. (Family 13: Parent)

In contrast, disengagement and dropout was considered to be a result of families not perceiving they needed FFT, lacking understanding of the purpose and potential benefits and/or not believing there had been any progress.

Either [they maybe] don't see the first stage [engagement] as being beneficial... they don't really see the purpose or why are we doing this ... [or they] don't see value in it. [That is when] I think they'll drop out. (Practitioner 3)

When the family is not seeing any change, disillusionment, disappointment ... a lot of the families haven't stuck at anything [before]. (Practitioner 6)

Previous engagement with support services and agencies, including schools, was described as underpinning families' expectations about FFT and their relationships with FFT practitioners. If families viewed previous involvement with services, including social work, as supportive this made engagement easier. In contrast, families who had previous negative experiences may be wary of getting involved in FFT. 
If you come in with a social worker who has a very, very good relationship with the family ... it makes it so much easier. If they have a tainted relationship ... then there's probably a natural tendency for them to assume that anything the social worker links them to could be, could deliver the same sort of service. (Practitioner 4)

Views of significant others. Support, or encouragement, from family, extended family and friends was another identified facilitator of engagement.
Ma mum pushin' me towards... me changin' ... she stood by me an'... helped me through' (Family 11: Adolescent, Male, 15-16y)
My best friend knows about it... she's also through social work... there's problems in her family ... she said that it was good because she [did] see me being kinda depressed, you know, before the family therapy. (Family 8: Adolescent, Female, 13-14y)

On the other hand, unsupportive others could act as a barrier.

$$
\begin{aligned}
& \text { Where you've got a family, a dad and a mum who [believe] this isn't gonna } \\
& \text { work... maybe external, extended family who are kind of "what you doing } \\
& \text { working with them" and all that kind of social work tag and it can be quite } \\
& \text { frustrating. (Practitioner 5) }
\end{aligned}
$$

Indeed, a minority of young people said that they did not tell their extended family or friends they were taking part in FFT for fear they would be judgmental or have negative perceptions of the therapy.

Psycho-social and cultural influences. Mental health problems or alcohol and substance misuse problems were considered to be barriers to engagement. These issues meant families might not be home for arranged visits, or the problem impacted on the individual's ability to 
participate in sessions. In addition, several practitioners reported on wider socio-economic and contextual factors impacting on families' ability to engage and maintain involvement with FFT.

Other things will take precedence over us at times you know, real kind of poverty issues, health issues... economic issues I think and housing issues. Things that are just beyond our influence (Practitioner 5)

Also, some practitioners felt that the local culture (west of Scotland) impacted on how comfortable some people were in talking about their emotions or problems they were experiencing, which might lead to disengagement. While others identified challenges when working with families from minority ethnic backgrounds, including families who have limited English or different cultural expectations in terms of parenting, child-rearing and gender roles.

A Sikh family and dad's from India and kind of limited English... mum is saying he is a typical Indian bloke, don’t expect any changes. (Practitioner 5) Again, this was viewed as impacting on acceptance and engagement with the intervention.

\section{Structure and delivery format of the FFT intervention}

The key themes to emerge in this category were: Flexibility is key; Work and homework can be fun; and, Home-delivery.

Flexibility is key. Practitioners and families spoke positively about FFT and its structure and delivery format. Practitioners felt that they had flexibility to tailor the intervention according to the needs of individual families.

One of the things I like about the model is that although there's a structure to it, within those sessions it is very much left up to the therapist how they deliver it so it allows you to bring your own style as well to it (Practitioner 3) 
Work and homework can be fun. The opportunity to use creative material and activities in sessions with the families was also highlighted as being an important facilitator.

It would be very bland and dry if you were just sitting talking at them ... so doing a communication skills thing so you will do the talking bit where you are explaining stuff ... but then you might do a little game... so they will have a little bit of fun with it and then ... afterwards [you] discuss why communication is important. (Practitioner 1)

This was reaffirmed in the narrative from families.

I had to say whatever it was on the paper, mum had to draw it... it helped a lot... knowing that we could communicate like that and... be happy when we were communicating... that made me feel better because I could say stuff to my mum. (Family 10: Adolescent, Female, 15-16y)

He was like am gonnae be giving you homework, and I was like, really?! Yea, I'm, I'm not in school... but it was very, very simple things like just spending time together, like watching a movie... (Family 10: Parent)

Home-delivery. A particularly important element for many families was the in-home delivery. There was a feeling that if families had to travel to participate in the intervention they would have been less likely to engage.

I think the three of us travelling to go somewhere... I don't think that would have happened. (Family 11: Parent)

That said, a small number of the family participants felt differently believing there was more chance of distraction in the home environment.

You don't concentrate as much in my house. If it was in a library I would concentrate more. (Family 7: Adolescent, Male, 13-14 years) 


\section{Organizational factors}

The FFT practitioners discussed organizational factors that facilitated or hindered their delivery of the intervention. The themes identified included: Buying-in to FFT; Team culture and credibility at the top; Building and honing skills; and Administration versus people contact.

Buying-in to FFT. Several practitioners talked about their own role and, in particular, about how practitioner 'compatibility' with, and belief in, FFT was important for successful implementation.

Recent research ... [shows] that working with the parent and child together has more successful outcomes. (Practitioner 1)

As an FFT worker there has to be a belief in what we're doing otherwise I don't think it would work. (Practitioner 2)

In addition, seeing change in families undertaking the intervention was described as a positive feedback loop, acting to evidence the effectiveness of FFT.

It's great! (laughs), cos I guess it just reinforces like that this does actually work erm and it makes you feel like you are doing something and you are changing, you might not be able to change the world but if you can change a small part of it. (Practitioner 3)

Team culture and credibility at the top. Several practitioners highlighted the importance of the personal qualities and experience of the individual leading the team of FFT practitioners. The current manager was described as being committed to and having a good understanding of FFT. They were also seen as having credibility because they managed their own caseload. These qualities appeared to be crucial in supporting others in the implementation process. In addition, many of the practitioners emphasized a supportive team culture as important for the successful implementation of FFT. 
Engagement in a team, to be able to work in a team, to engage in supervision... to be teachable really instead of being a closed book ... open communication with colleagues, being able to take on constructive criticism and challenge. (Practitioner 2)

Building and honing skills. On the whole, participation in the FFT clinical training program was viewed as supportive and strengths-based. The interactive elements of training, which involved role-play and reflecting on video recordings of their practice, were described as most helpful and the approach of the trainer was important.

Very supportive, very encouraging, very accepting, erm and sort of erm always focused on your positive contribution rather than erm what you maybe weren't doing as well. (Practitioner 4)

Although training was viewed positively, some of the practitioners highlighted areas where they would have welcomed more. In particular, they indicated a need for additional training in the administrative elements of implementation, such as the collection and recording of outcome data. Practitioners also spoke about the importance of follow-up training (after initial training) and of the value of learning through on-going practice experience.

In addition to training, on-going group supervision was presented as facilitating the acquiring and strengthening of FFT skills, and providing reassurance while highlighting areas for further development. This was helpful for practitioners planning sessions and for intervention adherence.

[Supervision] keeps you quite focused so there's not drift in terms of what you're doing with the family. Which is really important and it keeps you on your toes... (Practitioner 5) 
Supervision was particularly valued when practitioners encountered complex practice situations.

There's times when I'm just like, I can't think how to reframe this behavior... somebody will just give you a completely different perspective ... "have you ever thought about this?" (Practitioner 3)

The views expressed in relation to group supervision contrasted with views on individual occupational supervision (social work) that practitioners participated in as part of their organization's requirements. This was described as being more constraining and focused on performance rather than competency building. Some of the practitioners discussed the balancing of time to undertake supervision with the time needed to spend delivering the intervention. Also there appeared to be a need to discuss individual cases that was not always met.

Administration versus people contact. Finally, the practitioners discussed various administrative and workload issues that impacted the implementation process. Documenting and recording work with families and writing to other agencies was viewed, at times, demanding and time consuming, taking away from direct contact with families.

I think in my past work probably sixty percent of my work was direct contact with families and the other thirty, forty would have been my admin tasks... To begin with [with FFT] it was the complete opposite, and I was thinking this is awful, I'm not seeing anybody... (Practitioner 5)

Some felt that their current number of cases (eight families) could be challenging in terms of working effectively with families. Challenges related to time and case management included finding time to prepare and record sessions, traveling to the families' homes, and fitting in evening appointments. 


\section{DISCUSSION}

To date, this is the first study to have combined the views and experiences of practitioners, parents and adolescents to provide a more holistic account of key stakeholder views on the barriers and facilitators to the successful implementation of FFT. Overall both groups talked about FFT in a positive way, and both groups were able to share reflections on barriers and facilitators associated with their involvement in it.

\section{Barriers and facilitators to the successful implementation of FFT}

Effective partnerships. The referral process and pre-intervention contact with families in need were identified as critical points in the engagement decision-making process where barriers to participation in FFT could be overcome. This may be particularly important in service contexts, such as the present study, where FFT is offered on a voluntary basis. Linked to this, previous research has found no difference in outcomes between voluntary and mandated groups but, for both, the effectiveness of FFT was associated with the number of sessions attended, or level of engagement (Celinska, 2015).

In the current study, participants highlighted the need for there to be effective partnerships to facilitate the tripartite communication needed between agencies referring families to the service, the FFT practitioners, and the families. The first step was ensuring that partner agencies were aware of the existence of the FFT service, and that referring individuals had sufficient Knowledge and understanding of the potential benefits of the intervention so they could communicate this to families. Practitioners also had to ensure families in need were encouraged to participate in FFT without Feeling pressured to do so. Both practitioners and families articulated a view that a key facilitator of early engagement was the family knowing why they had been referred and how FFT might help their situation. This was a stage where families could be encouraged to believe that FFT had relevance within their own lives and had potential to help them change identified problems. 
While it was not possible, within the boundaries of this study, to explore reasons for disengagement in families who were offered but opted not to participate in FFT, it might be assumed that this was linked to them not perceiving a need for FFT or not seeing it as potential solution for the problems they faced. This supports evidence in the literature that perception of need is an important facilitator of engagement (Baydar, Reid, \& WebsterStratton, 2003; Dumas, Nissley-Tsiopinis, \& Moreland, 2007). As Katz et al. note "parents need to understand that there is a problem, believe that a service could help them" (Katz, La Placa, \& Hunter, 2007, p. 9). Indeed, even those families who reported Feeling pressured to start FFT described this within the context of knowing they 'needed something'.

Thus, the findings from this study, alongside existing literature, highlight the need for future implementation of FFT to embed tailored pre-intervention engagement strategies designed to address barriers and facilitators of initial engagement. This should include building the knowledge and understanding of referring individuals (Zazzali et al., 2008) and identifying ways of supporting families to recognize their need for support. It should also include demonstration of the ways in which FFT might bring benefit to each family; for example, case studies or testimonials from similar families might provide a contextualized illustration.

Therapeutic relationship. Supporting current research on factors influencing successful implementation of intervention programs (Forehand \& Kotcrick, 2002; Moran, Ghate, \& van der Merwe, 2004), both groups of stakeholders spoke about the importance of the therapeutic relationship for the facilitation of on-going engagement. As captured in the First steps in relationship building theme, this was initiated prior to the intervention starting in the first meeting between the practitioner and family. Families described FFT practitioners as adopting a personable, non-authoritative and informal approach. Indeed, providing an opportunity for rapport building between families and the practitioners they are to work with 
has previously been highlighted as important for engagement (Moran et al., 2004). In the current study, this may have been enhanced by conducting the first meeting, and subsequent intervention sessions, in the family home; this has be identified by others as a useful strategy for reducing barriers such as anxiety or hostility (Ghate \& Ramella, 2002).

When talking about the therapeutic relationship during the delivery of the intervention, practitioners and families described a partnership that was underpinned by Trust and honesty, where families felt that their individual needs were being respected and where they could speak openly. As in other studies (Forehand \& Kotchick, 2002; Ghate, Shaw, \& Hazel, 2000), this partnership approach appeared to promote a sense of control amongst family members who felt listened to and acknowledged as experts in their own lives. Strategies such as the FFT practitioner being open to contact with the family in-between sessions and following completion of FFT facilitated the maintenance of the therapeutic relationship and engagement. Imporantly, the approach adopted by practitioners mirrored the strength-based relational focus promoted by the FFT model (Alexander et al., 2013) and provides evidence that practitioners were engaging with families in a way that was appropriate to the theoretical underpinnings of the intervention. Positive engagement during the early stages of FFT, Engagement and Motivation, has the potential to open the way for use of a range of family therapeutic treatment strategies. These, in turn, allow FFT therapists to implement cognitive and behavioral techniques matched to the target family's values, relational function and other important factors known to influence successful change (Alexander et al., 2013).

Individual, family and social factors. A number of themes emerged that highlighted individual, family and social factors that had the potential to act as barriers and facilitators to engagement during the 3 month delivery period. As noted above, while recognition of the problems faced and a belief that support was required facilitated initial engagement, experiencing positive change during the intervention was considered an important facilitator 
of continued engagement. In contrast, a number of barriers to engagement were identified, including: Psycho-social and cultural influences, such as mental health problems, substance and/or alcohol misuse; negative Views of significant others about the family participating in the intervention; and, pre-conceptions about support services and therapy. It is not possible with the available data to review these factors further but previous literature suggests that they can cause families to be resistant to intervention or, alternatively, mean that engagement strategies are not sufficiently sensitive to the family's needs (Docherty, Stott, \& Kinder, 2004; Koerting et al., 2013). Clearly these two different scenarios require different solutions. For example, work conducted in the Netherlands has demonstrated that contextualized engagement strategies can be used to encourage high risk families (e.g., where mental health problems are present) to participate in FFT (Breuk et al., 2006). While further research is needed to explore this in more depth, the findings from this study illustrate a need for staff who deliver FFT to be sensitized and responsive to the individual needs of families when working to overcome barriers to engagement.

Intervention structure and delivery. Three key themes emerged in relation to intervention structure and delivery; Flexibility is key; Work and homework can be fun; and, Homedelivery. These themes capture the views of the majority of adolescents and parents/grandparents about resources, activities and strategies that enhanced their engagement with, and the effect of, the FFT intervention. In addition to being enjoyable, these activities were described as helping families to develop good communication and listening skills, perhaps strengthening the therapeutic relationship. This reinforces a widely held view that practitioners can best involve participants in interventions through an interactive approach, rather than one where the practitioner dominates (Katz et al., 2007). There is clear acknowledgement that for interventions to be effective they require to meet the needs of the families they serve, both in terms of the contextual sensitivity of treatment transport and the 
tailoring of individual sessions for individual families (Fixsen et al., 2005; Koerting et al., 2013); however, ways of achieving this require careful consideration to ensure that intervention fidelity remains optimal.

Organizational factors. Practitioners were asked to share their views on the organizational factors that impacted on the implementation of FFT. In the theme Buying-in to FFT, it was interesting that many of the practitioners described their own role in implementation rather than focusing on barriers/facilitators that result from organizational infrastructure. Although not explicit in the data, this may have been a result of the practitioners having been part of the FFT team since its inception, which could have engendered a sense of ownership of the implementation process in a way that might not be the case for individuals joining teams where the initial phases of implementation are completed. This may also have been reinforced by the fact they were a team of staff with a dedicated FFT workload who, therefore, did not need to balance FFT delivery with other competing demands.

Linked to this, there was a view that implementation was enhanced when practitioners had existing qualifications or training that were compatible with FFT and when they had a belief in the effectiveness of the model. Supporting research shows implementation of evidence-based innovation to be more successful if the intervention is compatible with current practice and if practitioners believe the intervention will bring benefit (Aarons \& Palinkas, 2007).

Practitioners spoke about the importance of a supportive team culture and the instrumental role of the team leader in overcoming some of the barriers to implementation. This resonates with implementation science literature advocating the need for an influential leader who shows commitment to the intervention and implementation process and who has rapport with staff members (Fixsen et al., 2005; Milhalic, Fagan, Irwin, Ballard, \& Elliott, 2004). Indeed, teams where there are individuals who can provide modeling of expectations 
and demonstrate the viability of the implementation model are more likely to achieve implementation success (Breuk et al., 2006).

Training and supervision, as a means to Building and honing skills, were presented by practitioners as facilitating their delivery of FFT, mirroring assumptions in the literature that supervision assists intervention staff to apply newly gained knowledge and skills in practice and contributes to preparedness in delivery (Fixsen et al., 2005). This is important given evidence that the effectiveness of FFT is contingent on, at least in part, therapist adherence to the model (Graham et al., 2014; Sexton \& Turner, 2010). That said, practitioners were able to articulate ways in which they felt both training and supervision could be enhanced; for example, additional training to support the administration of the intervention and the collection of outcome data. This type of feedback is important for training providers who need to ensure that on-going support is (perceived as) responsive to the particular needs of the practitioners because perceived lack of post-training knowledge and/or skill has previously been identified as a barrier to subsequent delivery of family-focused interventions (Sanders, Prinz, \& Shapiro, 2009). In addition, the request for problem-focused supervision supports research in other domains showing that practitioners welcome the opportunity to discuss issues that will enhance practice (McPherson, Sanders, Schroeter, Troy, \& Wiseman, 2016). Therefore, in addition to ensuring that practitioners are skilled to undertake administrative tasks efficiently, training and supervision should be tailored to promote better understanding of the need for administration (e.g., outcome recording) to ensure optimal implementation of FFT and other evidence-based interventions (Durlak \& Dupre, 2008).

In summary, participants in the current study described a range of barriers and facilitators to successful implementation of FFT that crossed the full journey, from referral to the service to participation in the FFT sessions. These were linked to: the ways in which the service partnered with other agencies; the therapeutic relationship between the practitioners 
and the families participating in the intervention; other individual, family and social factors that had the potential to disrupt the engagement process; the structure and delivery format of FFT; and, organization factors linked that facilitated or hindered the practitioners' delivery of the intervention.

\section{Limitations}

It is important that the interpretation of the findings, the conclusions drawn, and the recommendations made are considered within the limitations of the study. Specifically, given the qualitative nature of the study, it cannot claim to be representative of all families offered FFT; however, the sampling strategy was designed to ensure diversity in the participants and thus in the views and experiences shared. Given the focus on experiences related to participation in FFT, only families who had completed the intervention took part in this study. Therefore, the findings do not illuminate the experiences of those who chose not to participate in FFT or those families who were considered by practitioners to be too 'high risk' to participate in the research. Further research is required to bridge this gap in knowledge to ensure that implementation strategies can be designed to target families with different engagement needs. In addition, future research endeavors need to consider other stakeholders who may hold different views on the implementation process; for example, agencies who refer families for FFT, service managers and FFT supervisors.

\section{Conclusion}

This is the first study to seek views on barriers and facilitators to the successful implementation of FFT from the people delivering and those in receipt of it. It is also one of the first to seek the views of young people who have completed a family-based intervention. As such, this study makes a significant contribution to the FFT literature and the wider literature on the implementation of evidence-based interventions for families. A key priority for global public health is the implementation of early interventions to prevent poor life- 
course outcomes (World Health Organization, 2014) and FFT has been offered as one such evidence-based intervention for adolescents displaying behavioral problems (Littell et al., 2007). Complementing the literature evidencing effect, the findings from this current study suggest that FFT is considered to be an acceptable, appropriate and feasible intervention by two key stakeholder groups; the families in receipt of it and the practitioners who deliver it. Therefore, with attention to the barriers and facilitators highlighted by this study, FFT should be considered an important part of the evidence-based toolkit available to improve the lives of adolescents and their families.

\section{References}

Aarons, G. A., \& Palinkas, L. A. (2007). Implementation of evidence-based practice in child welfare: Service provider perspectives. Administration and Policy in Mental Health and Mental Health Services Research, 34(4), 411-419. doi:10.1007/s10488-007-0121-3

Alexander, J .F. (2011). Functional Family Therapy Clinical Training Manual. Washington: FFTLLC.

Alexander, J. F., \& Parsons, B. V. (1973). Short-term behavioral intervention with delinquent families: Impact on family process and recidivism. Journal of Abnormal Psychology, 81(3), 219-225. doi:10.1037/h0034537

Alexander, J. F., Waldron, H. B., Robbins, M. S., \& Neeb, A. A. (2013). Functional Family Therapy for adolescent behavior problems. Washington: American Psychological Association.

American Psychiatric Association (2013). Diagnostic and Statistical Manual of Mental Disorders (DSM-5), Fifth Edition. Arlington, VA: American Psychiatric Association Publishing. 
Baldwin, S. A., Christian, S., Berkeljon, A., \& Shadish, W. R. (2012). The effects of family therapies for adolescent delinquency and substance abuse: A meta-analysis. Journal of Marital and Family Therapy, 38(1), 281-304. doi:10.1111/j.1752-0606.2011.00248.x

Bhaskar, R. A. (1989). Reclaiming reality: A critical introduction to contemporary philosophy. Sussex: Harvester Press.

Baydar, N., Reid, M. J., \& Webster-Stratton, C. (2003). The role of mental health factors and program engagement in the effectiveness of a preventative parenting program for Head Start mothers. Child Development, 74, 1433-1453.

Braun, V., \& Clarke, V. (2006). Using thematic analysis in psychology. Qualitative Research in Psychology, 3(2), 77-101. doi:10.1191/1478088706qp063oa

Brauner, C. B., \& Stephens, C. B. (2006). Estimating the prevalence of early childhood serious emotional/behavioral disorders: Challenges and recommendations. Public Health Reports, 121(3), 303-310.

Breuk, R. E., Sexton, T. L., Van Dam, A., Disse, C., Doreleijers, T. A. H., Slot, W. N., \& Rowland, M. K. (2006). The implementation and the cultural adjustment of functional family therapy in a Dutch psychiatric day-treatment center. Journal of Marital and Family Therapy, 32(4), 515-529. doi:10.1111/j.1752-0606.2006.tb01625.x

Casey, B., McPherson, K. E., \& Kerr, S. (2016). Functional Family Therapy: Perceptions and experiences of key stakeholders in Glasgow. Glasgow: Glasgow Caledonian University.

Celinska, K. (2015). Effectiveness of Functional Family Therapy for mandated versus nonmandated youth. Juvenile and Family Court Journal, 66(4), 17-27.

Celinska, K., Cheng, C., \& Virgil, N. J. (2015). Youth and parental perspectives on the functional family therapy programme. Journal of Family Therapy, 37(4), 450-470. doi:10.1111/14676427.12051 
Docherty, P., Stott, A., \& Kinder, K. (2004). Delivering services to hard to reach families in on track areas: Definition, consultation and needs assessment. London: Home Office.

Dumas, J. E., Nissley-Tsiopinis, J., \& Moreland, A. D. (2007). From intent to enrollment, attendance, and participation in preventative parenting groups. Journal of Child and Family Studies, 16(1), 1-26.

Durlak, J.A. \& Dupre, E.P. (2008). Implementation matters: A review of research on the influence of implementation on program outcomes and the factors affecting implementation. American Journal of Community Psychology, 41, 327-350.

Fixsen, D. L., Blase, K. A., Duda, M. A., Naoom, S. F., \& Van Dyke, M. K. (2010). Implementation of evidence-based treatments for children and adolescents: Research findings and their implications for the future. In J. Weisz, \& A. Kazdin (Eds.), Evidence-based psychotherapies for children and adolescents (2nd ed.). New York: Guilford Press.

Fixsen, D. L., Naoom, S. F., Blase, K. A., \& Friedman, R. M. (2005). Implementation research: A synthesis of the literature. Florida: University of South Florida.

Forehand, R., \& Kotchick, B. A. (2002). Behavioral parent training: Current challenges and potential solutions. Journal of Child and Family Studies, 11(4), 377-384. doi:10.1023/A:1020913422609

Ghate, D., \& Ramella, M. (2002). Positive parenting: The national evaluation of the youth justice board's parenting programme. London: Policy Research Bureau.

Ghate, D., Shaw, C., \& Hazel, N. (2000). Fathers and family centres: Engaging fathers in preventive services. York: Joseph Rowntree Foundation.

Graham, C., Carr, A., Rooney, B., Sexton, T., \& Satterfield, A. R. W. (2014). Evaluation of functional family therapy in an Irish context. Journal of Family Therapy, 36, 20-38. 
Hill, J. (2002). Biological, psychological and social processes in the conduct disorders.

Journal of Child Psychology and Psychiatry, and Allied Disciplines, 43(1), 133-164. doi:10.1111/1469-7610.00007

Houston, S. (2001). Beyond social constructionism: Critical realism and social work. British Journal of Social Work, 31(6), 845-861. doi:10.1093/bjsw/31.6.845

Katz, I., La Placa, V., \& Hunter, S. (2007). Barriers to inclusion and successful engagement of parents in mainstream services. York: Joseph Rowntree Foundation.

Koerting, J., Smith, E., Knowles, M. M., Latter, S., Elsey, H., McCann, D. C., . . . SonugaBarke, E. J. (2013). Barriers to, and facilitators of, parenting programmes for childhood behaviour problems: A qualitative synthesis of studies of parents' and professionals' perceptions. European Child \& Adolescent Psychiatry, 22(11), 653-670. doi:10.1007/s00787-013-0401-2

Liu, J. (2004). Childhood externalizing behavior: Theory and implications. Journal of Child and Adolescent Psychiatric Nursing, 17(3), 93-103. doi:10.1111/j.17446171.2004.tb00003.x

Littell, J. H., Winsvold, A., Bjørndal, A., \& Hammerstrøm, K. T. (2007). Functional family therapy for families of youth (age 11-18) with behaviour problems. Cochrane Database of Systematic Reviews 2007, Issue 2. Art. No.: CD006561. DOI: 10.1002/14651858.CD006561.

McEvoy, P. \& Richards, D. (2003). Critical realisim: A way forward for evaluation research in nursing? Journal of Advanced Nursing, 43(4), 411-420.

McPherson, K. E., Kerr, S., McGee, E., Morgan, A., Cheater, F. M., McLean, J., \& Egan, J. (2014). The association between social capital and mental health and behavioural problems in children and adolescents: An integrative systematic review. BMC Psychology, 2: 7. doi:10.1186/2050-7283-2-7 
McPherson, K. E., Sanders, M. R., Schroeter, B., Troy, V., \& Wiseman, K. (2016). Acceptability and feasibility of peer assisted supervision and support for intervention practitioners: A Q-methodology evaluation. Journal of Child and Family Studies, 25(3), 720-732.

Mihalic, S., Fagan, A., Irwin, K., Ballard, D., \& Elliott, D. (2004). Blueprints for violence prevention. Washington: Office of Juvenile Justice and Delinquency Prevention.

Moffitt, T. E., Caspi, A., Harrington, H., \& Milne, B. J. (2002). Males on the life-coursepersistent and adolescence- limited antisocial pathways: Follow-up at age 26 years. Development and Psychopathology, 14(1), 179-207. doi:10.1017/S0954579402001104

Moran, P., Ghate, D., \& van der Merwe, A. (2004). What works in parenting support? A review of the international evidence. London: Department for Education and Skills. NICE (2013). Antisocial behaviour and conduct disorders in children and young people: Recognition, intervention and management. London: NICE.

NICE (2011). Alcohol-use disorders: Diagnosis, assessment and management of harmful drinking and alcohol dependence. London: NICE.

NVivo (2012). NVivo qualitative data analysis software. QSR International Pty Ltd. Version 10, 2012.

Oliver, C. (2012). Critical realist grounded theory: A new approach for social work research. British Journal of Social Work, 42(2), 371-387. doi:10.1093/bjsw/bcr064

Patten, S. B., Wilkes, T. C. R., Williams, J. V. A., Lavorato, D .H., el-Guebaly, N., Wild, T. C., Colman, I., \& Bulloch, A.G.M. (2016). Childhood adversity and subsequent mental health status in adulthood: screening for associations using two linked surveys. Epidemiology and Psychiatric Sciences, 25(2), 160-170. 
Price, J.M., Chiapa, A., \& Walsh, N.E. (2013). Predictors of externalizing behavior problems in early elementary-aged children: the role of family and home environments. Journal of Genetic Psychology, 174(4), 464-71.

Proctor, E., Silmere, H., Raghavan, R., Hovmand, P., Aarons, G., Bunger, A., Griffey, R., \& Hensley, M. (2011). Outcomes for implementation research: Conceptual distinctions, measurement challenges, and research agenda. Administration and Policy in Mental Health, 38(2), 65-76.

Rapport, M. D., Denney, C. B., Chung, K., \& Hustace, K. (2001). Internalizing behavior problems and scholastic achievement in children: Cognitive and behavioral pathways as mediators of outcome. Journal of Clinical Child Psychology, 30, 536-551.

Robbins, A. S., Alexander, J. F., Turner, C. W., \& Hollimon, A. (2016). Evolution of Functional Family Therapy as an evidence-based practice for adolescents with disruptive behavior problems. Family Process, 55(3), 543-557. doi:10.1111/famp.12230

Sanders, M. R., Prinz, R. J., \& Shapiro, C. J. (2009). Predicting utilization of evidence-based parenting interventions with organizational, service-provider and client variables. Administration and Policy in Mental Health and Mental Health Services Research, 36(2), 133-143. doi:10.1007/s10488-009-0205-3

Sexton, T., \& Turner, C. W. (2010). The effectiveness of Functional Family Therapy for youth with behavioral problems in a community practice setting. Journal of Family Psychology, 24(3), 339-348. doi:10.1037/ a0019406

Sholevar, E., Baron, D. A., Aussetts, M. A., \& Spiga, R. (2010). Treatment adherence in family therapy with youth as a predictor of time to arrest. American Journal of Family Therapy, 38(1), 1-15. doi:10.1080/ 01926180902754695

Stratton, P (2005). Report on the evidence base of systemic family therapy. Association for Family Therapy. 
Tonge B. (2007). The psychopathology of children with intellectual disabilities. In Bouras N., Holt, G., (Eds.), Psychiatric and behavioral disorders in intellectual and developmental disabilities (pp. 93-112). New York: Cambridge University Press.

von Sydow, K., Retzlaff, R., Beher, S., Haun, M.W., \& Schweitzer, J. (2013). The efficacy of systemic therapy for childhood and adolescent externalizing disorders: A systematic review of 47 RCT. Family Process, 52(4), 576-618. doi: 10.1111/famp.12047

Washington State Institute for Public Policy (2016). Benefit-cost technical documentation. Olympia, WA: Author.

Weisz, J. R., \& Kazdin, A. E. (Eds.). (2010). Evidence-based psychotherapies for children and adolescents (2nd ed.). New York: Guilford Press.

Woolfenden, S. R., Williams, K. \& Peat, J. K. (2002). Family and parenting interventions for conduct disorder and delinquency: a meta-analysis of randomised controlled trials. Archives of Disease in Childhood, 86, 251-256. doi:10.1136/adc.86.4.251

World Health Organization (2014). Health for the world's Adolescents: A second chance in the second decade summary. Geneva: World Health Organization. Available at http://apps.who.int/adolescent/seconddecade/files/1612_MNCAH_HWA_Executive_Summary.pdf

Zazzali, J. L., Sherbourne, C., Hoagwood, K. E., Greene, D., Bigley, M. F., \& Sexton, T. L. (2008). The adoption and implementation of an evidence based practice in child and family mental health services organizations: A pilot study of Functional Family Therapy in New York State. Administration and Policy in Mental Health and Mental Health Services Research, 35, 38-49. doi: 10.1007/s10488-007-0145-8 